\title{
Research of the Vehicle Load Control System Integration Device
}

\author{
Jun $\mathrm{Li}^{1}$, Yanzhao $\mathrm{Su}^{1}$, Jinli Xie ${ }^{1}$, Yangjiao $\mathrm{Xu}^{1} \& \mathrm{Lei} \mathrm{J}^{1,2}$ \\ ${ }^{1}$ Chongqing Jiaotong University, Chongqing, China \\ ${ }^{2}$ Great Wall Motor Incorporated Company, Baoding, China \\ Correspondence: Jun Li, Mechantronics and Automotive Engineering, Chongqing Jiaotong University, 400074, \\ Chongqing, China. Tel: 86-138-8390-1379. E-mail: xuyangjiao1986@gmail.com
}

\author{
Received: April 9, 2014 Accepted: May 2, 2014 Available online: May 8, 2014 \\ doi:10.11114/set.v1i2.384 \\ URL: http://dx.doi.org/10.11114/set.v1i2.384
}

\begin{abstract}
With the gradual development of economy, the scale of transportation industry continues to expand. The problem of overload or overrun in the vehicle transport has emerged. Therefore, how simply and conveniently to know the vehicle load and how to effectively limit overload has become a key issue. This paper demonstrates the feasibility of vehicle load control system from the above problems. Through the pressure sensors installed in the vehicle suspension, the single-chip microprocessor receives the information transmitted by the pressure sensors, and calculates the total weight of the vehicle load; if overweight, the single-chip microprocessor will send commands to the ignition system, to stop the ignition system working. Its purpose is to improve vehicle safety and effectively reduce heavy workload of the vehicle detection station to improve their work efficiency.
\end{abstract}

Keywords: vehicle load, pressure sensors, computer control, the ignition system

\section{Introduction}

Vehicle load control system integration device can detect conveniently vehicle load to prevent overloading of vehicle and improve vehicle safety. And it can effectively reduce heavy work of the vehicle load testing station and improve work efficiency. Transport sectors achieve intelligent vehicle traffic and unified management to save the country a lot of manpower and material resources by achieving real-time business management summary and checking the vehicle load data (Meng, 2010; Li, 2010) .

The value lies in providing safe and convenient travel for the users. As the device is working in the loading process, it can ensure to prevent vehicle overloading; In the process of driving, the drivers don't have to worry about being fined due to overload syndrome; ensure the personal safety of driving. At the same time the system is designed to save the national highway maintenance fees and to ensure the safety of people's lives and property (Li, 2010); it can solve the problem of the damage of highway Bridges, can also travel to provide a more humanized service life for the people, and for the country's economic construction contribute a strength.

\section{Overall Structure of the System}

The overall structure of the system is shown in Figure 1. And the system is divided into information acquisition module, information conversion module and information execution module.

Information acquisition module can collect the vehicle load information accurately and reliably and transit the real-time information to the computer processor efficiently and safely. Using the single-chip microprocessor, the information conversion module can achieve the amplification of the signals and the analysis and transmission of the information in harsh environment (Xiao, 2009; Jin et al., 2009). The information execution module can receive various commands which are sent by the computer processor and execute tasks efficiently.

\subsection{Information Acquisition Module}

There is a laminated spring between the car body and the axle, shown in Figure 2. The damping property of laminated spring is well and the force and deformation curve are shown in Figure 3. The deformation energy which is consumed by frictional heat cased the nonlinear of the vehicle unloading curve while the loading force and deformation are linear and the elastic coefficient is a constant $\mathrm{C}$. This makes the circuit design feasible. Measure the force according to the spring deformation and thus measure the weight of the goods indirectly. Install the strain gauge pressure sensors in the 
laminated spring (Figure 2) and install the MPM282 OEM pressure sensors at the front axle of the vehicle and the midpoint of the rear axle (Jin et al., 2009).

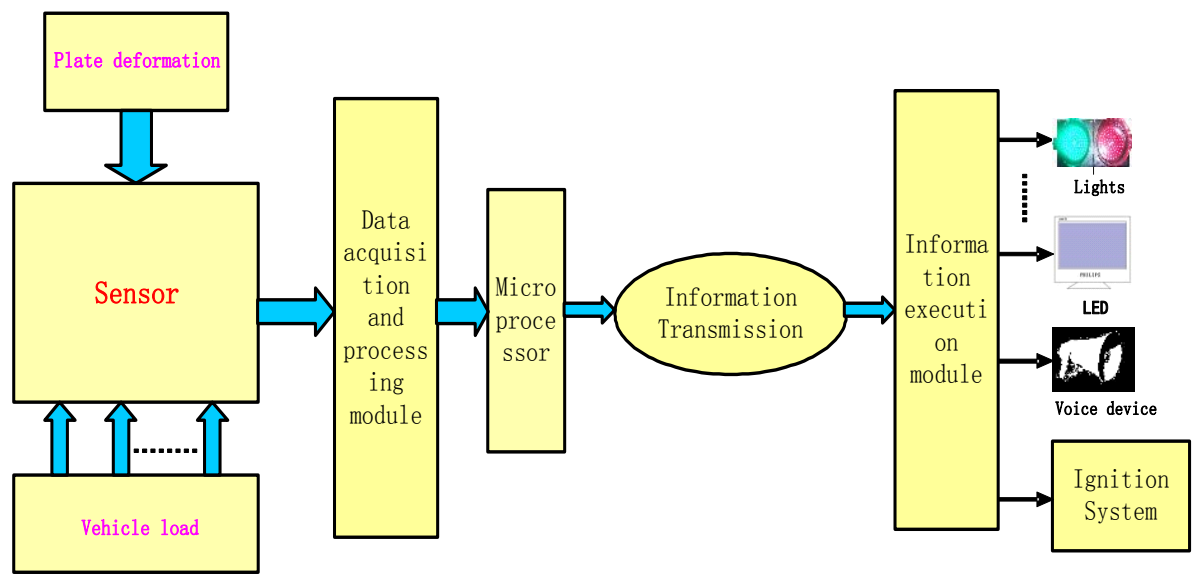

Figure 1. Overall structure of vehicle load control system

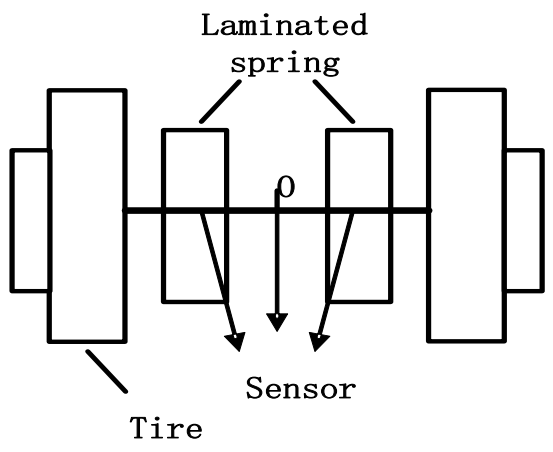

Figure 2. Laminated spring diagram

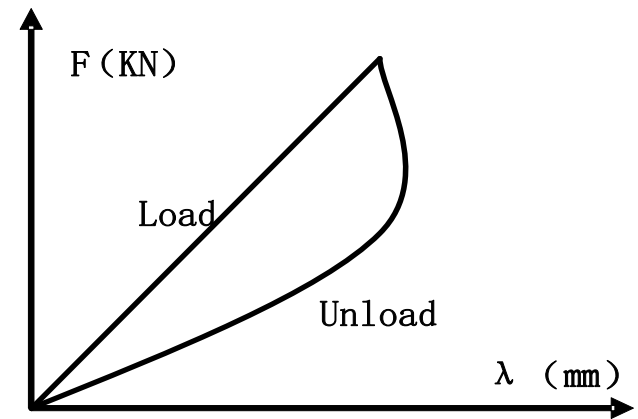

Figure 3. Laminated spring force deformation curve

Gather the vehicle load pressure signal by pressure sensors (Jing, 2007). The pressure collected (which can be translated into the physical load) is converted into an electrical signal that can gather the vehicle load information real-time and transit the information to the computer processor accurately, reliably, efficiently and safely(Chen et al., 2005). The load control system to the circuit diagram of the input section is shown in Figure 4.

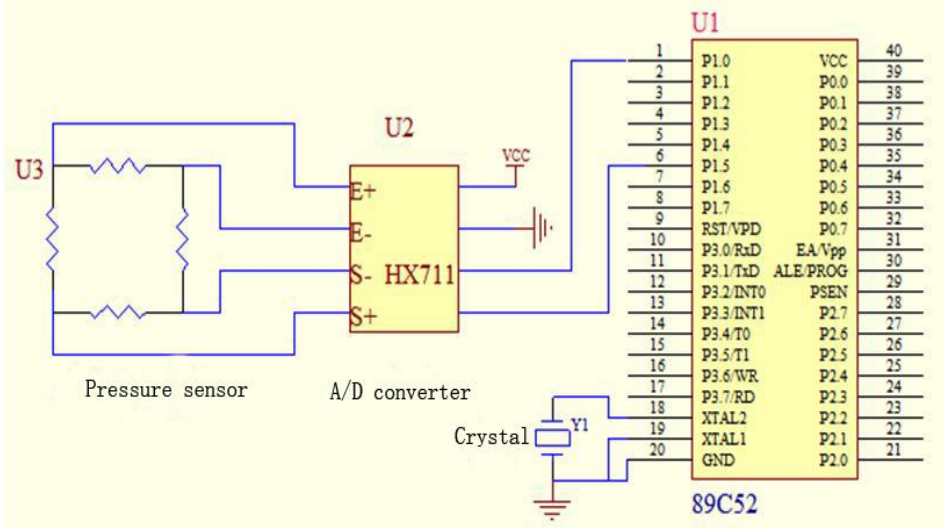

Figure 4. Load control system to the circuit diagram of the input section

\subsection{Information Conversion Module}

Using single-chip microprocessor, the information conversion module can receive variety of amplified signals collected by pressure sensors in harsh environment, turn the analog to digital with $\mathrm{A} / \mathrm{D}$ and $\mathrm{D} / \mathrm{A}$ converters, calculate the total load, and then transmit the data to the LED monitor (Bi, 2003). According to different vehicle load, the single-chip microprocessor will choose whether to send instruction to the ignition system or not, to control the ignition system. In 
addition, the single-chip microprocessor will control the lights flashing and the voice device alarm or not (Zhao, 2010). The single-chip microprocessor overall structure is shown in Figure 5.

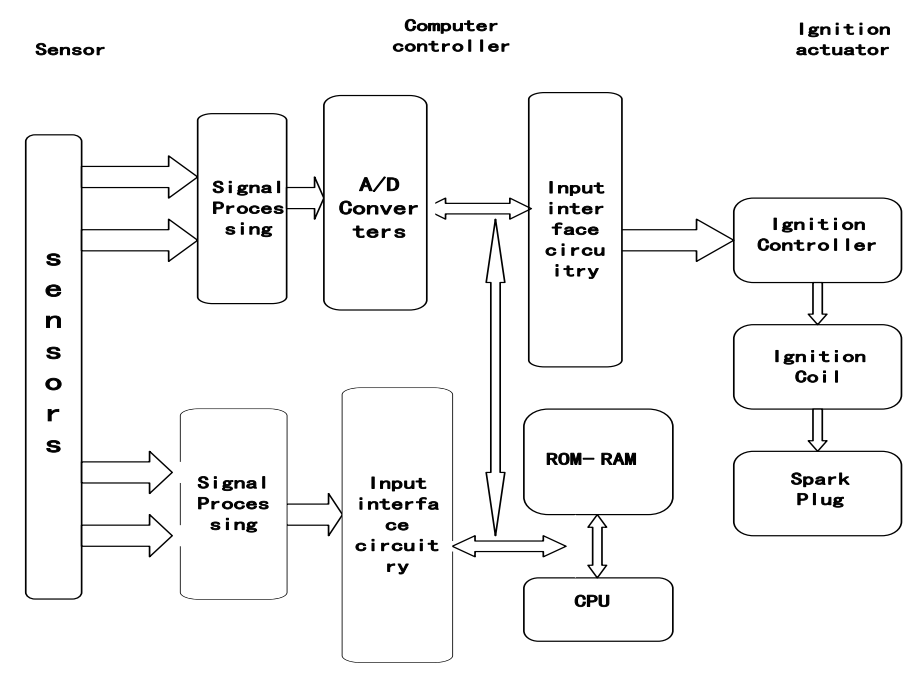

Figure 5. The single-chip microprocessor overall structure

\subsection{Information Execution Module}

Information execution module can receive all kinds of instructions sent by microprocessor and execute a mission effectively and quickly. The execution of the main components includes ignition system, LED display, voice device and signal device. The load control system to output section of a circuit is shown in Figure 6.

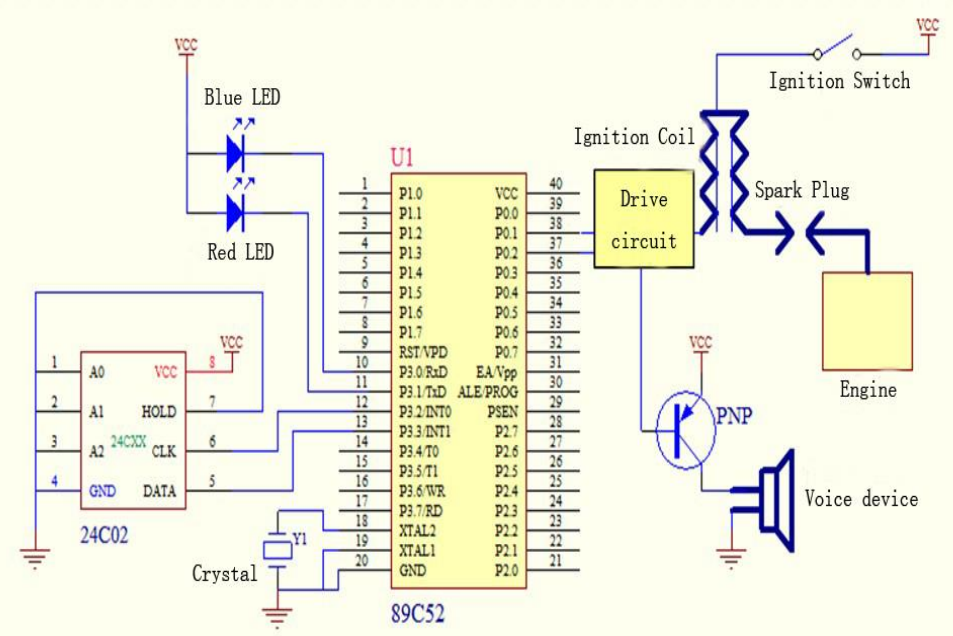

Figure 6. Load control system to output section of a circuit

\section{The Design of System Software}

After the pressure sensor measures the load of the vehicle, the load controller will identify the level amplitude of the signal sent by pressure sensor and calculate the load(Gao, et al., 2006). The trigger which the working principle is shown in Figure 7 is used for changing the level.

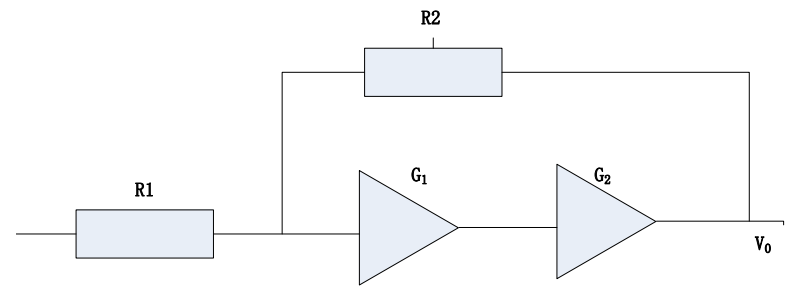

Figure 7. The trigger working principle diagram 
The threshold voltage of the inverter is :

$$
V_{i 1}=\frac{R_{2}}{R_{1}+R_{2}} V_{i}+\frac{R_{2}}{R_{1}+R_{2}} V_{o}
$$

When $V_{i}=0, G_{1}$ is off as well as $G_{2}$ is on, the output $V_{0}=0 V, V_{1}=0 V$. With the gradually increasing of input $V_{1}$, the output $\mathrm{V}_{0}=0 \mathrm{~V}$ as long as $\mathrm{Vil}$ is under $\mathrm{V}_{\mathrm{th}}$. The circuit will have the following positive feedback process when $\mathrm{V}_{\mathrm{i}}=\mathrm{V}_{\mathrm{th}}$ : $V_{i 1} \uparrow \rightarrow V_{o 1} \uparrow \rightarrow V_{o} \uparrow$ The forward threshold voltage is :

$$
V_{T+}=\left(1+\frac{R_{1}}{R_{2}}\right) V_{t h}
$$

When $V_{i t}>V_{\text {th }}, V_{0}=V_{d d}$. When $\mathrm{Vi}$ continues to rise to its maximum value and then begin decrease, the circuit will have the following positive feedback process when $\mathrm{V}_{\mathrm{i}}=\mathrm{V}_{\mathrm{th}}: V_{i 1} \downarrow \rightarrow V_{o 1} \uparrow \rightarrow V_{o} \downarrow$. The negative threshold voltage is :

$$
V_{T-}=\left(1-\frac{R_{1}}{R_{2}}\right) V_{t h}
$$

Hysteresis voltage is:

$$
\Delta V_{T}=V_{T+}-V_{T-}=2 \frac{R_{1}}{R_{2}} V_{t h}
$$

Adjust the resistance of $R_{1}$ and $R_{2}$ to change the hysteresis voltage. When the forward threshold voltage $V_{t+}$ is equal to the negative threshold voltage $\mathrm{V}_{\mathrm{t}}$, the electrical signal sent by pressure sensor can be amplitude discriminating.

When the vehicle is overloaded, the signal sent by pressure sensor is higher than the set level afterwards the trigger will restrict the ignition system; while the signal sent by pressure sensor is lower than the set level, the trigger will put a level to make the ignition system work properly.

\section{The System Work Flow Chart}

The system is consisted of pressure sensor, voice device, signal lights, monitor and ignition system and its work flow chart is shown in Figure 8. The signal light is green without an overweight while the light begin to flash; when the weight is up to the standard weight the speaker would start to prompt(Zhang, 2004; An, 2010); the light will turn to red when the vehicle is overweight.

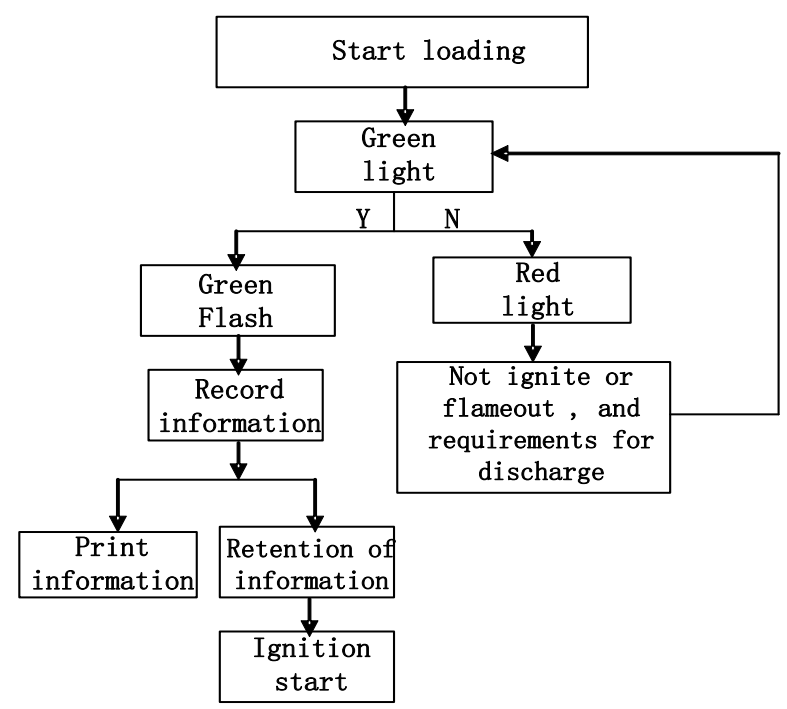

Figure 8. The system work flow chart

\section{Conclusion}

The system is simple and convenient to be able to know the vehicle load and can solve the problem of vehicle overloading effectively. It has improved some shortcomings of the detection overloading devices that are in low 
efficiency, large and moving inconveniently. While it reduces the incidence of traffic accidents and the serious damage caused by overload vehicles on the road, people's lives and property are protected. As vehicles will be moving in the direction of the future development of intelligent, some research results in this paper can provide a reference for future improvement of vehicle safety driving techniques.

\section{References}

An, Z. J. (2010). Pressure Sensor Automatic Verification System. Metrology \& Measurement Technique. 26-27. http://www.cqvip.com/QK/95027A/200812/1000555513.html

Bi, S. W. (2003). Coal Mine Using Electronic Weighing Apparatus Selection Method. China Coal Technology, 32-33. http://www.cqvip.com/QK/94487A/2003004/8944236.html

Chen, J. R., \& Ma, T. F. (2005). Automobile Structure. Beijing: 295-301.

Gao, H. J., James, L., \& Wang, C.H. (2006). Multi-objective control of vehicle active suspension systems via load-dependent controllers. Journal of Sound and Vibration, 5-8. http://www.sciencedirect.com/science/article/pii/S0022460X05002920

Jin, W., \& Wu, Z. Q. (2009). 51 Series MCU Development of the LED Display Technology. Beijing: 18-23.

Jing, L. (2007). The Overload Control of Vehicles. East Road, 11-12. http://www.docin.com/p-69242698.html

Li, Y. (2010). The Development and Application of Foreign Cars Overload Control Technology. Highway Construction and Transportation, 191-196. http://www.cqvip.com/Main/Detail.aspx?id=10327501

Meng, X. Z. (2010). Automobile Transportation Safety Management Laws and the Standardized Construction in Heilongjiang Province. Communications Science and Technology Heilongjiang, 193-194. http://www.cnki.com.cn/Article/CJFDTotal-HLJJ201009137.html

Xiao, Y. Y. (2009). The Design of Traffic Light Circuit. The Motor Information, 131-132. http://www.cnki.com.cn/Article/CJFDTotal-JDXX200930073.html

Zhang, Y. W. (2004). Introduction to Digital Weighing Sensor Technology Present Situation and the Trend.China Weighing Instrument Association, 9-11. http://www.cnki.com.cn/Article/CJFDTotal-HEQI200401000.html

Zhao, Q. Y. (2010). The Overload Sensing Technology Based Car Refused to Start Design. Sensor World, 18-19. http://www.cnki.com.cn/Article/CJFDTotal-CGSJ201006006.html

\section{(cc) $\mathrm{Br}$}

This work is licensed under a Creative Commons Attribution 3.0 License. 